Sciendo

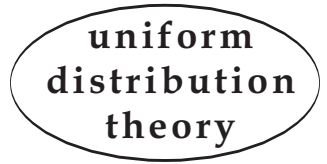

DOI: $10.2478 /$ udt-2021-0011

Unif. Distrib. Theory 16 (2021), no.2, 129-136

\title{
AO. UNIV.-PROF. DR. REINHARD WINKLER (1964-2021) AN OBITUARY
}

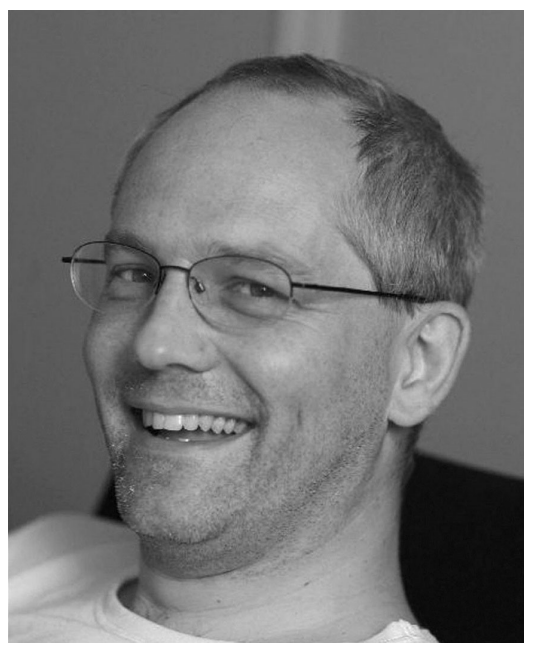

The Vienna University of Technology, the Faculty of Mathematics and Geoinformation and the Institute of Discrete Mathematics and Geometry mourn the death of ao. Univ.-Prof. Reinhard Winkler. We are deeply saddened to announce that Reinhard Winkler passed away on October 13, 2021.

Reinhard Winkler was born on July 5, 1964 in Vienna, where he also attended the grammar school BGRG 8 in Albertgasse. He studied Technical Mathematics at the Vienna University of Technology, besides Mathematical Logic, Philosophy and Musicology at the University of Vienna.

He completed his $\mathrm{PhD}$ in 1988 with the thesis "Analytische und algebraische Beiträge zur Theorie der Gleichverteilung" (Analytical and Algebraic Contributions to the Theory of Equal Distribution) under Prof. Robert Tichy, followed by his habilitation in 1994. Until 2003 he was a half-time employee at both the

(C) 2021 BOKU-University of Natural Resources and Life Sciences and Mathematical Institute, Slovak Academy of Sciences.

(c) (1) $\odot$ Licensed under the Creative Commons BY-NC-ND 4.0 International Public License. 
Academy of Sciences and the Vienna University of Technology, and from 2003 a full-time employee as ao.Univ.Prof. at the Institute for Discrete Mathematics and Geometry at the Vienna University of Technology.

His scientific opus touches several areas of mathematics, including number theory, algebra and topology; among others, he led a subproject in the "National Research Network Analytic Combinatorics and Probabilistic Number Theory" of the FWF. In 2000 he was awarded the "Edmund and Rosa Hlawka Prize of the Austrian Academy of Sciences".

Reinhard Winkler was an extremely dedicated university teacher, who was particularly concerned with the basic mathematical education of both engineers and teacher candidates. In addition to his teaching activities, he regularly participated actively in the didactics days for mathematics teachers, wrote numerous didactic publications in which he emphasized the interrelation of different areas of mathematics, and was a member of the Didactics Commission of the Austrian Mathematical Society.

In addition, the popularization of mathematics was a major concern of his. He was active in math.space for many years and was one of the founders of TUFor Math (Forum Mathematik der TU Wien).

Reinhard Winkler was passionate about classical music and was an accomplished piano player. He was a versatile conversationalist, whether it was about art, literature, politics, philosophy, ethics, psychology or, of course, mathematics. His many friends and acquaintances appreciated him as a kind and empathetic person.

We will miss him very much.

Our special sympathy goes to his life person Eva and his family.

The funeral took place on Thursday, November 4, 2021 at Neustift Cemetery in Vienna.

Martin Goldstern and Michael Drmota

\section{List of publication of Reinhard Winkler}

[1] Winkler, R.: Two results in uniform distribution theory. Anz. Österreich. Akad. Wiss. Math.-Natur. Kl. 124 (1987), 57-59.

[2] Eigenthaler, G.-Winkler, R.: Commutative composition semigroups of polynomials, Contributions to General Algebra 6 (1988), 89-101.

[3] Tichy, R.-WinkleR, R.: Gleichverteilung in hyperkomplexen Systemen, Österreich. Akad. Wiss. Math.-Natur. Kl. Sitzungsber. II, 197 (1988), no. 8-10, 417-432. 


\section{OBITUARY}

[4] Winkler, R.: Some constructive examples in uniform distribution theory on finite sets and normal numbers, Anz. Österreich. Akad. Wiss. Math.-Natur. Kl. 126 (1989), 1-8.

[5] Drmota, M.-Tichy, R.-Winkler, R.: Completely uniformly distributed sequences of matrices. In: Number-theoretic analysis (Vienna, 1988-89), Lecture Notes in Math. Vol. 1452, Springer-Verlag, Berlin, 1990, pp. 43-57.

[6] Winkler, R.: Strong Weyl property. In: Number-theoretic analysis (Vienna, 1988-89), Lecture Notes in Math. Vol. 1452, Springer-Verlag, Berlin, 1990, pp. 208-220.

[7] Tichy, R.-WinkleR, R.: Uniform distribution preserving mappings, Acta Arith. 60 (1991), no. 2, 177-189.

[8] Tichy, R.-WinkleR, R.: Bemerkungen über Pseudozufallszahlen und deren Anwendung zur Komposition von Walzern, Österreich. Akad. Wiss. Math.-Natur. Kl. Sitzungsber. II, 200 (1991), no. 1-10, 53-63.

[9] Winkler, R.: Algebraic endomorphisms of LCA-groups that preserve closed subgroups, Geom. Dedicata, 43 (1992), 307-320.

[10] Winkler, R.: Polynomial approximation on locally compact abelian groups, Studia Sci. Math. Hungar. 28 (1993), no. 1-2, 135-144.

[11] Winkler, R.: On maximal abelian groups of maps, J. Austral. Math. Soc. Ser. A 55 (1993), no. 3, 414-420.

[12] Winkler, R.: Some remarks on pseudorandom sequences, Math. Slovaca 43 (1993), no. 4, 493-512.

[13] Grabner, P.-Tichy, R.-Winkler, R.: On the stability of the quotients of successive terms of linear recurring sequences. In: Number Theoretic and Algebraic Methods in Computer Science, Moscow (A. van der Poorten, I. Shparlinski, H. G. Zimmer, eds.) (1993), pp. 185-192,

[14] Drmota, M.-Winkler, R.: $s(N)$-uniform distribution modulo 1, J. Number Theory 50 (1995), 213-225.

[15] Winkler, R.: Polynomial approximation on locally compact abelian groups II. Studia Sci. Math. Hungar. 30 (1995), 265-274.

[16] Schmeling, J.-Winkler, R.: Typical dimension of the graph of certain functions, Monatsh. Math. 119 (1995), 303-320.

[17] Winkler, R.: Monotonic functions and an inequality of Myerson on point distributions, Indag. Math. (N.S.) 6 (1995), no. 2, 247-255. 
[18] Winkler, R.: A simple Hausdorff space which is Lindelöf, first contable, CCC but not separable, Anz. Österreich. Akad. Wiss. Math.-Natur. Kl. 133 (1996), 23-25.

[19] Winkler, R.: On the distribution behaviour of sequences, Math. Nachr. 186 (1997), 303-312.

[20] Winkler, R.: Sets of block structure and discrepancy estimates, Journal de Théorie des Nombres de Bordeaux 9 (1997), 337-349.

[21] Winkler, R.: Distribution preserving sequences of maps and almost constant sequences on finite sets, Monatsh. Math. 126 (1998), 161-174.

[22] Frisch, S.-PAŠtékA, M.-Tichy, R. F.-Winkler, R.: Finitely additive measures on groups and rings, Rend. Circ. Mat. Palermo, Ser. II, 48 (1999), 323-340.

[23] WinkLeR, R.: Distribution preserving transformations of sequences on compact metric spaces, Indag. Math. (N.S.) 10 (1999), no. 3, 459-471.

[24] WinkLER, R.: Topological automorphisms of modified Sierpiński gaskets realize arbitrary finite permutation groups, Topology Appl. 101 (2000), 137-142.

[25] Goldstern, M.-Schmeling, J.-Winkler, R.: Metric, fractal dimensional and Baire results on the distribution of subsequences, Math. Nachr. 219 (2000), 97-108.

[26] Schmeling, J.-Szabó, E.-Winkler, R.: Hartman and Beatty bisequences. In: Proceedings of the International Conference held in Graz, Austria, August 30 to September 5, 1998, Algebraic Number Theory and Diophantine Analysis, Walter de Gruyter, Berlin, New York, 2000, pp. 405-421.

[27] WinkleR, R.: How much must an order theorist forget to become a topologist? In: Proceedings of the Vienna Conference, June 3-6, 1999, Contributions to General Algebra Vol. 12 (2000), Verlag Johannes Heyn, Klagenfurt, pp. 419-433.

[28] Winkler, R.: The order theoretic structure of the set of P-sums of a sequence, Publ. Math. Debrecen 58 (2001), no. 3, 467-490.

[29] Winkler, R.: Wie macht man 2 aus 1? Das Paradoxon von Banach-Tarski. Didaktikhefte der Österreichischen Mathematischen Gesellschaft, Schriftenreihe zur Didaktik der Mathematik der Höheren Schulen 33 (2001), 166-196.

[30] WinkleR, R.: Ergodic group rotations, Hartman and Kronecker sequences, Monatsh. Math. 135 (2002), 333-343.

[31] WinkleR, R.: Maximal abelian group actions on the ordered real line and their digital representations, Aequationes Math. 66 (2003), 204-231. 


\section{OBITUARY}

[32] Glass A. M. W.-Rachůnek, J.-Winkler, R.: Functional representations and universals for $M V$ - and GMV-algebras, Tatra Mt. Math. Publ. 27 (2003), 91-110.

[33] Glass A. M. W.-Winkler, R.: Rooted wreath products, J. Algebra 273 (2004), 489-506.

[34] Steineder, C.-Winkler, R.: Complexity of Hartman sequences, J. Théor. Nombres Bordeaux 17 (2005), 347-357.

[35] Winkler, R.: Die Mathematik zum Leuchten, Tanzen und Klingen bringen eine längst überfällige Polemik zur Bildungsdiskussion, "Die Presse" (daily newspaper), 3.9.2005.

[36] Beiglböck, M.-Steineder, C.-Winkler, R.: Sequences and filters of characters characterizing subgroups of compact abelian groups, Topology Appl. 153 (2006), 1682-1695.

[37] Winkler, R.: Von Nutzen, Wert und Wesen mathematischer Bildung. In: Bildung zwischen Nutzen und Notwendigkeit, Lit-Verlag, Münster-Berlin-Hamburg-London-Wien, 2006.

[38] WinkleR, R.: Mathematik im Banne der Schönheit: Zur Veranstaltungsreihe "math.music" im Wiener math. space, Die Presse (daily newspaper), 28 (2007), no. 2, under the title: "Aphrodite, Beethoven und die Infinitesimalrechnung".

[39] Winkler, R.: Hartman sets, functions and sequences - a survey, Adv. Stud. Pure Math. 49 (2007), 517-543.

[40] Winkler, R.: Sinn und Unsinn des Rechnens im Mathematikunterricht, Didaktikhefte der ÖMG, 39 (January 2007), 155-165.

[41] Goldstern, M.-Schmeling, J.-Winkler, R.: Further Baire results on the distribution of subsequences, Unif. Distrib. Theory, 2 (2007) no. 1, $127-149$.

[42] Albrecher, H.-Drmota, M.-Goldstern, M.-Winkler, R.: Robert F.Tichy: 50 years - The unreasonable effectiveness of a number theorist, Unif. Distrib. Theory, 2 (2007), no.1, 151-160.

[43] Winkler, R.: Was ist Mathematik? - Ein subjektiver Zugang. English translation by Otmar Binder: What is mathematics?-a subjective approach. In: The Language of Science - ISSN 1971-1352 - Monza: Polimetrica, 2007.

[44] WinkleR, R.: Wir zählen bis drei - und sogar darüber hinaus. Didaktikhefte der ÖMG 40 (January 2008), 129-141. 
[45] WinkleR, R.: Nachhaltigkeit von Mathematikunterricht durch Förderung der Phantasie. In: Professionalität und Professionalisierung. Einige aktuelle Fragen und Ansätze der universitären LehrerInnenbildung (Ilse Schrittesser, ed.), Peter Lang GmbH Internationaler Verlag der Wissenschaften, Frankfurt am Main, 2009, pp. 179-205.

[46] Winkler, R.-Mozart, P.: Galois - Symmetrie und Asymmetrie als Vermittlerinnen zwischen Form und Inhalt. In: The catalogue of the exhibition Oskar Putz-Bilder und Farbkonzepte, January 15-25, 2009, Künstlerhaus, Wien (Christine Janicek, ed.), Gezeiten Verlag \& Kommunikation, Wien, 2009, pp. 41-68.

[47] Winkler, R.: Die reellen Zahlen sind anders, Didaktikhefte der ÖMG 41 (January 2009), 140-153.

[48] Akiyama, S.-Dorfer, G.-Thuswaldner, J.-Winkler, R.: On the fundamental group of the Sierpinski gasket, Topology Appl. 156 (2009), $1655-1672$.

[49] Maresch, G.-Winkler, R.: Compactifications, Hartman functions and (weak) almost periodicity, Dissertationes Math. 461 (2009), 1-72.

[50] WinkleR, R.: Erinnerungen an den wissenschaftlichen Großvater (In the memory of Prof. Edmund Hlawka (1916-2009)), Internat. Math. Nachrichten 211 (August 2009), 1-26.

[51] Winkler, R.: Was leistet der Mathematikunterricht in der Schule und was soll bzw. kann er leisten? Report from a panel discussion. In: The joint conference of the German and Austrian mathematical societies DMV and ÖMG, Graz, September 24, 2009. Internat. Math. Nachrichten 212 (December 2009), 23-28.

[52] Christof, E.-Mateus-Berr, R.-Sattlberger, E.- Skone, J.Tamussino, C.-Winkler, R. And students: Maths goes design-design goes maths. (Documentation of a joint project 2007-2010 of three universities: Universität für angewandte Kunst, Universität Wien, Technische Universität Wien and Math. space, Universität für Angewandte Kunst), 2009/10.

[53] Winkler, R.: Logischer und mengentheoretischer Formalismus-Ärgernis, und sonst nichts? Didaktikhefte der ÖMG 42 (January 2010), 102-117.

[54] Winkler, R.: Auf welche mathematische Schulbildung möchte die Universität aufbauen können? Stellungnahme der TU, Wien, 2010.

[55] Winkler, R.: A little topological counterpart of Birkhoff's ergodic theorem, Unif. Distrib. Theory 5 (2010), no. 1, (the volume dedicated to the memory of Professor Edmund Hlawka (1916-2009)), 157-162. 
[56] Winkler, R.: Der Organismus der Mathematik; mikro-, makro- und mesoskopisch betrachtet. In: Mathematik verstehen-philosophische und didaktische Perspektiven (Markus Helmerich, Katja Lengnink, Gregor Nickel, Martin Rathgeb, eds.), Vieweg + Teubner Verlag, 2011, pp. 59-70.

[57] Winkler, R.: Das Maß aller Dinge aus mathematischer Sicht-zu den Grundideen der Integralrechnung. Didaktikhefte der ÖMG 43 (January 2011), $146-160$.

[58] Winkler, R.: Mathematik: Die unauflösliche Verflochtenheit von Fachwissenschaft und Didaktik. In: Best Spirit: Best Practice, Lehramt an Österreichischen Universitäten. Braumüller Verlag, 2011.

[59] Winkler, R.: Im Anfang war die Exponentialfunktion, Didaktikhefte der ÖMG 44 (January 2012), 98-109.

[60] Beiglböck, M.-Winkler, R.: Endre Szemeredi: Ein mathematisches Universum in kombinatorischem Gewande, Internat. Math. Nachr. 221 (December 2012), 21-38.

[61] Winkler, R.: Stochastik-ein Fest der Unabhängigkeit, Didaktikhefte der ÖMG 45 (2012), 122-136.

[62] Winkler, R.: Mathematische Prozesse im Widerstreit. In: Mathematik im Prozess. Philosophische, Historische und Didaktische Perspektiven. (Martin Rathgeb, Markus Helmerich, Ralf Krömer, Katja Lengnink, Gregor Nickel, eds.) Springer Spektrum, Wiesbaden, 2013, pp. 89-100.

[63] Dorfer, G.-Thuswaldner, J.-Winkler, R.: Fundamental groups of one-dimensional spaces, Fundamenta Mathematicae 223 (2013), 137-169.

[64] Winkler, R.: Dynamische Systeme als Chance für den Schulunterricht, Schriftenreihe zur Didaktik der Mathematik der Österreichischen Mathematischen Gesellschaft ÖMG 46 (2013), 108-122.

[65] Winkler, R.: Die Geburt der Mathematik aus den Bedingungen der Musik, Schriftenreihe zur Didaktik der Mathematik der Österreichischen Mathematischen Gesellschaft ÖMG 47 (2014), 108-122.

[66] Winkler, R.: Drei Versuche, eine kulturell bedeutende Universität zu werden (Three attempts to become a culturally significant university). In: Die Technik und die Musen-Kunst und Kultur im Umfeld der Technischen Universität Wien (Technology and the Muses - Art and Culture around the TU Wien. (Juliane Mikoletzky, ed.) (2016), pp. 28-51. (Part of Technik für Menschen - 200 Jahre Technische Universität Wien (Sabine Seidler, ed.), Böhlau Verlag, Wien, Köln, Weimar.) 


\section{OBITUARY}

[67] WinkLER, R.: Gedanken zu einer zeitgemäßen mathematischen Grundausbildung in den Ingenieurwissenschaften (Thoughts on modern mathematical basic education in the engineering sciences). In: Die Fakultät für Mathematik und Geoinformation (Michael Drmota, ed.), The Faculty of Mathematics and Geoinformation, 2016, pp. 97-100. (Part of Technik für Menschen - 200 Jahre Technische Universität, Wien (Sabine Seidler, ed.), Böhlau Verlag, Wien, Köln, Weimar.)

[68] Winkler, R.: Zentralmatura-quo vadis? Schriftenreihe zur Didaktik der Mathematik der Österreichischen Mathematischen Gesellschaft ÖMG, 49 (2016), 131-144.

[69] Winkler, R.: Mathematik als zentraler Teil des Projektes Aufklärung auf breiter Front. In: Mathematik und Gesellschaft. Historische, philosophische und didaktische Perspektiven. (Gregor Nickel, Markus Helmerich, Ralf Krömer, Katja Lengnink, Martin Rathgeb, eds.) Springer Spektrum, 2018, pp. 99-106.

[70] Winkler, R.: Zentralmatura in der Sackgasse? Internat. Math. Nachrichten 237 (April 2018), 27-58.

[71] Winkler, R.: Das TU Forum Mathematik in Wien - Gedanken zur Popularisierung von Mathematik, Internat. Math. Nachrichten 238 (August 2018), $45-58$.

[72] Hell, T.-Kaltenbacher, B.-Winkler, R.-Woess, W.: ÖMG-Studierendentreffen und Early Student Award, Internat. Math. Nachrichten 239 (December 2018), 25-34.

[73] Winkler, R.: Der Grenzwert-Zentralbegriff der Analysis. Schriftenreihe zur Didaktik der Mathematik der Österreichischen Mathematischen Gesellschaft ÖMG 51 (2018), 97-111.

[74] Winkler, R.: Abstraktion in Mathematik und Mathematikunterricht. Schriftenreihe zur Didaktik der Mathematik der Österreichischen Mathematischen Gesellschaft ÖMG 52 (2019), 113-126. 